\title{
Species delimitation based on mtDNA genes suggests the occurrence of new species of Mesocestoides in the Mediterranean region
}

\author{
Antonio Varcasia ${ }^{1 *+}$, Daria Sanna ${ }^{2 \dagger}$, Marco Casu ${ }^{1}$, Samia Lahmar ${ }^{3}$, Giorgia Dessì ${ }^{1}$, Anna Paola Pipia ${ }^{1}$, \\ Claudia Tamponi ${ }^{1}$, Gabriella Gaglio ${ }^{4}$, Gabriela Hrčková ${ }^{5}$, Domenico Otranto ${ }^{6}$ and Antonio Scala ${ }^{1}$
}

\begin{abstract}
Background: This study is the first contribution to the molecular taxonomy of Mesocestoides spp. from domestic and wild carnivores in the Mediterranean area. A total of 13 adult worms and 13 larval stages of Mesocestoides spp. were collected from domestic and wild carnivore hosts in Italy and Tunisia. Samples collected in the Slovak Republic were used as comparative samples from outside the Mediterranean. The genes cytochrome c oxidase subunit 1 (cox1) and NADH dehydrogenase subunit 1 (nad1) of the mitochondrial genome were used as molecular markers to investigate the presence of cryptic Mesocestoides species in the area analysed.

Results: Results were consistent in showing three well-supported clusters of Mesocestoides spp. in southern Italy and Tunisia, which were strongly divergent from Mesocestoides litteratus, M. corti and M. lineatus. High levels of genetic variation and no evidence of geographical structuring was found between the clusters.

Conclusions: Considering the low dispersal capability of the intermediate hosts of Mesocestoides spp., the lack of geographical structuring among the Mediterranean regions could be due to a high potential for dispersion of the definitive hosts. This study provides a foundation for future formal descriptions of new species of the genus Mesocestoides in the Mediterranean area.
\end{abstract}

Keywords: Mesocestoides, Dog, Cat, Fox, Genetic structuring, Species delimitation, Mediterranean region

\section{Background}

The genus Mesocestoides (Cyclophyllidea, Mesocestoididae) includes parasites with unique peculiarities in many aspects of their biology, which have yet to be revealed [1]. Two intermediate hosts are likely required for the completion of the Mesocestoides life-cycle [2], with the first larval stage developing in coprophagous arthropods, and the second (i.e. tetrathyridium) in a wide variety of hosts (e.g. rodents, amphibians, reptiles and birds) [1, 3, 4]. Adult Mesocestoides worms have been recorded in up to $13.8 \%$ of cats, $26.5 \%$ of dogs, $70 \%$ of jackals and $81.8 \%$ of foxes $[2,5-8]$. The latter species seem to be the most

\footnotetext{
* Correspondence: varcasia@uniss.it

${ }^{+}$Antonio Varcasia and Daria Sanna contributed equally to this work.

1 Dipartimento di Medicina Veterinaria, Università di Sassari, Sassari, Italy

Full list of author information is available at the end of the article
}

important definitive hosts for these parasites as confirmed by a recent paper that highlighted a prevalence of $84.1 \%$ in red foxes from Poland [9]. Wild and domestic carnivores could also serve as second intermediate hosts [10] since tetrathyridium larvae can multiply asexually by longitudinal fission, penetrate the intestinal wall, invade the peritoneal cavity of the hosts and eventually cause life-threatening peritonitis [11-13]. In addition, Mesocestoides spp. are potentially zoonotic, being reported in human infections (at least 27 cases) following the consumption of raw or undercooked snake, chicken and wild game viscera $[14,15]$.

The distribution of Mesocestoides species is not well delineated due to their high degree of phenotypic plasticity, which hinders a clear morphological delineation of the species [5]. Furthermore, identification at the species level is not possible for the larval stages from intermediate

(c) The Author(s). 2018 Open Access This article is distributed under the terms of the Creative Commons Attribution 4.0 International License (http://creativecommons.org/licenses/by/4.0/), which permits unrestricted use, distribution, and 
hosts [16] or also when parasites are recovered incomplete, as gravid proglottids. This might have led to the failure of correct species identification in the past [5]. Seven species of Mesocestoides have been recorded in Europe (in the Czech Republic, Slovak Republic and Spain) $[4,5,16,17]$ with $M$. litteratus and $M$. lineatus being the most widely distributed species. Although adults of Mesocestoides lineatus and Mesocestoides litteratus may be differentiated morphologically by subtle differences in the structure of the cirrus-sac, the number of testes and the position of the ovary and vitellarium $[18,19]$, a biomolecular confirmation of the morphological diagnosis is not exhaustive [20-25]. To date, information regarding intermediate and paratenic hosts of $M$. litteratus and $M$. lineatus in natural conditions is lacking [4].

Recently, a clear genetic distinction of M. lineatus and $M$. litteratus has been investigated in specimens displaying minor differences in male and female reproductive organs of worms collected from red foxes in Slovak Republic [5]. Although these parasites are common in the Mediterranean region [2, 6, 13, 26, 27], very few studies have investigated the taxonomy and the molecular characterization of Mesocestoides in this area.

In the present study, several individuals of Mesocestoides spp. from different hosts in southern Italy and Tunisia have been studied by sequencing of the cytochrome $c$ oxidase subunit 1 ( $\operatorname{cox} 1$ ) and NADH dehydrogenase subunit 1 ( $\mathrm{nad} 1$ ) mitochondrial genes, in order to shed new light on the possible occurrence of new genetic variants and/or species. Molecular species delimitation methods were therefore applied. Furthermore, cox 1 and nad 1 sequences from Mesocestoides litteratus and M. lineatus specimens collected in Slovak Republic and deposited in the Parasitic Worms Collection at the Natural History Museum, London, were obtained in this study to be used as comparative material.

\section{Methods}

The study was carried out on a total of 13 adult worms and 13 larval stages (tetrathyridia) of Mesocestoides spp. These were collected between 2014 and 2017 from animals (dogs and cats) referred for clinical visits and elective surgeries or recovered during necropsy (dogs and foxes) at the Veterinary Teaching Hospitals of the Universities of Sassari, Bari, Messina and Naples (Italy), and at the National School of Veterinary Medicine, Sidi Thabet (Tunisia). Details on hosts, parasites and sampling locations are reported in Table 1 and in Fig. 1. Morphological identification of parasites to the genus level was performed, when possible, according to available keys [5]. Fragments from five adult individuals of M. litteratus and one of $M$. lineatus found in foxes from Slovak Republic were also included in the study [5] in order to perform phylogenetic analysis. These specimens were identified according to Skrjabin [18], mounted on slides and deposited in the Parasitic Worms Collection at the Natural History Museum, London under the accession numbers BMNH 2011.2.2.1-3 and BMNH 2011.2.2.19-20, and have been used as comparative/reference material, in this study focused on parasites of the Mediterranean region.

DNA was extracted using a commercial PureLink ${ }^{\circ}$ Genomic DNA Mini Kit (Invitrogen, Carlsbad, California, USA) according to manufacturer's instructions. Partial fragments of the mitochondrial $\operatorname{cox} 1$ and $n a d 1$ genes were amplified by polymerase chain reaction (PCR) following previously described protocols [5, 28-30]. PCR products were purified using a Nucleospin Gel and PCR Clean Up kit (Macherey-Nagel, Düren, Germany) and sent to an external sequencing service (Eurofins Genomics, Ebersberg bei München, Germany). Sequence alignments were performed using BioEdit 7.2.5 [31] and deposited in the GenBank database under the accession numbers MH463491-MH463537 (see Table 1 and Additional file 1: Table S1 and Additional file 2: Table S2 for details). The levels of genetic polymorphism within parasites from the Mediterranean region were assessed using DnaSP 5.10 [32]. A median-joining network [33] was constructed using Network 5.0.0.3 (www.fluxus-engineering.com) to infer the genetic relationships among the haplotypes. A 95\% statistical parsimony network analysis was performed using TCS 1.21 [34], aimed at searching for possible disconnections between groups of individuals. The occurrence of genetic structure among samples was investigated by the Bayesian model-based clustering algorithm implemented in Baps 6 [35]. Each analysis was performed 10 times with a vector of values (1-10) for K each with 5 replicates.

For the phylogenetic analysis, two enlarged datasets for both $\operatorname{cox} 1$ and nad1 markers were built by aligning the sequences obtained in the present study with all comparable sequences available on GenBank for $M$. litteratus, M. lineatus and $M$. corti/M. vogae $[5,36,37]$ (see Figs. 4 and 5 for details). Two sequences from Italy (Tuscany, GenBank: JQ740884; and Sicily, GenBank: KU821650) attributed to Mesocestoides spp. were also included in the cox 1 dataset. A sequence of Echinococcus multilocularis from GenBank was used as the outgroup (Figs. 4, 5 and Table 1). The cox 1 gene dataset included 26 sequences obtained in the present study (see Table 1 for details) and 40 from GenBank. For the nad1 gene, the fragment analysed for phylogeny was restricted to $202 \mathrm{bp}$ in order to obtain an overlapping segment between our sequences and those from GenBank. The new nad 1 dataset included 21 sequences obtained in the present study (see Table 1 for details) and 25 sequences from GenBank.

In order to test the phylogenetic signal [38] and the adequacy of taxonomic coverage, the likelihood-mapping 
Table 1 Data collection and list of the specimens and the sequences included in the analyses

\begin{tabular}{|c|c|c|c|c|c|c|c|c|c|c|}
\hline \multirow[t]{2}{*}{ Sample ID } & \multirow[t]{2}{*}{ Host } & \multirow[t]{2}{*}{ Parasite stage } & \multirow[t]{2}{*}{ Isolated from } & \multirow[t]{2}{*}{ Origin } & \multirow[t]{2}{*}{ Morphological ID } & \multirow[t]{2}{*}{ Sampling site } & \multirow[t]{2}{*}{ Region } & \multirow[t]{2}{*}{ Country } & \multicolumn{2}{|l|}{ GenBank ID } \\
\hline & & & & & & & & & $\cos 1$ & nad1 \\
\hline APU01 & Dog & Tetrathyridium & Peritoneum & Surgery & Mesocestoides sp. & Bari & Apulia & Italy & MH463494 & MH463520 \\
\hline APU02 & Dog & Tetrathyridium & Peritoneum & Surgery & Mesocestoides sp. & Bari & Apulia & Italy & MH463495 & MH463521 \\
\hline APU04 & Dog & Tetrathyridium & Peritoneum & Surgery & Mesocestoides sp. & Bari & Apulia & Italy & MH463496 & MH463522 \\
\hline APU05 & Dog & Tetrathyridium & Liver & Surgery & Mesocestoides sp. & Bari & Apulia & Italy & MH463497 & MH463523 \\
\hline APU06 & Dog & Tetrathyridium & Intestine & Faecal sample & Mesocestoides sp. & Bari & Apulia & Italy & MH463498 & MH463524 \\
\hline APU07 & Dog & Tetrathyridium & Peritoneum & Surgery & Mesocestoides sp. & Bari & Apulia & Italy & MH463499 & MH463525 \\
\hline APU08 & Dog & Tetrathyridium & Peritoneum & Surgery & Mesocestoides sp. & Bari & Apulia & Italy & MH463500 & MH463526 \\
\hline APU09 & Dog & Tetrathyridium & Peritoneum & Surgery & Mesocestoides sp. & Bari & Apulia & Italy & MH463501 & MH463527 \\
\hline CAM01 & Dog & Tetrathyridium & Peritoneum & Surgery & Mesocestoides sp. & Napoli & Campania & Italy & MH463505 & MH463530 \\
\hline SAR01 & Cat & Adult worm & Intestine & Faecal sample & Mesocestoides sp. & Villacidro & Sardinia & Italy & MH463502 & na \\
\hline SARO2 & Dog & Tetrathyridium & Peritoneum & Surgery & Mesocestoides sp. & Sassari & Sardinia & Italy & MH463503 & MH463528 \\
\hline SAR03 & Fox & Adult worm & Intestine & Necropsy & Mesocestoides sp. & Mamoiada & Sardinia & Italy & MH463504 & MH463529 \\
\hline SIC01 & Cat & Tetrathyridium & Peritoneum & Surgery & Mesocestoides sp. & Messina & Sicily & Italy & MH463491 & MH463517 \\
\hline SIC02 & Dog & Tetrathyridium & Peritoneum & Surgery & Mesocestoides sp. & Messina & Sicily & Italy & MH463492 & MH463518 \\
\hline SICO3 & Cat & Tetrathyridium & Peritoneum & Surgery & Mesocestoides sp. & Messina & Sicily & Italy & MH463493 & MH463519 \\
\hline TUN01 & Dog & Adult worm & Intestine & Necropsy & Mesocestoides spp. & Sidi Thabet & $\begin{array}{l}\text { Sidi } \\
\text { Thabet }\end{array}$ & Tunisia & MH463506 & na \\
\hline TUN02 & Dog & Adult worm & Intestine & Necropsy & Mesocestoides spp. & Sidi Thabet & $\begin{array}{l}\text { Sidi } \\
\text { Thabet }\end{array}$ & Tunisia & MH463507 & MH463531 \\
\hline TUN03 & Dog & Adult worm & Intestine & Necropsy & Mesocestoides spp. & Sidi Thabet & $\begin{array}{l}\text { Sidi } \\
\text { Thabet }\end{array}$ & Tunisia & MH463508 & MH463532 \\
\hline TUN04 & Dog & Adult worm & Intestine & Necropsy & Mesocestoides spp. & Sidi Thabet & $\begin{array}{l}\text { Sidi } \\
\text { Thabet }\end{array}$ & Tunisia & MH463509 & MH463533 \\
\hline TUN05 & Dog & Adult worm & Intestine & Necropsy & Mesocestoides spp. & Sidi Thabet & $\begin{array}{l}\text { Sidi } \\
\text { Thabet }\end{array}$ & Tunisia & $\mathrm{MH} 463510$ & na \\
\hline TUN06 & Dog & Adult worm & Intestine & Necropsy & Mesocestoides spp. & Sidi Thabet & $\begin{array}{l}\text { Sidi } \\
\text { Thabet }\end{array}$ & Tunisia & MH463511 & na \\
\hline $\mathrm{SRO} 1^{\mathrm{a}}$ & Fox & Adult worm & Intestine & Necropsy & $\begin{array}{l}\text { Mesocestoides } \\
\text { litteratus }\end{array}$ & Košice & Košice & $\begin{array}{l}\text { Slovak } \\
\text { Republic }\end{array}$ & MH463512 & MH463534 \\
\hline $\mathrm{SRO} 2^{\mathrm{a}}$ & Fox & Adult worm & Intestine & Necropsy & $\begin{array}{l}\text { Mesocestoides } \\
\text { litteratus }\end{array}$ & Košice & Košice & $\begin{array}{l}\text { Slovak } \\
\text { Republic }\end{array}$ & MH463513 & MH463535 \\
\hline $\mathrm{SRO3}^{\mathrm{a}}$ & Fox & Adult worm & Intestine & Necropsy & $\begin{array}{l}\text { Mesocestoides } \\
\text { litteratus }\end{array}$ & Košice & Košice & $\begin{array}{l}\text { Slovak } \\
\text { Republic }\end{array}$ & $\mathrm{MH} 463514$ & MH463536 \\
\hline $\mathrm{SRO} 4^{\mathrm{a}}$ & Fox & Adult worm & Intestine & Necropsy & $\begin{array}{l}\text { Mesocestoides } \\
\text { lineatus }\end{array}$ & Košice & Košice & $\begin{array}{l}\text { Slovak } \\
\text { Republic }\end{array}$ & MH463515 & MH463537 \\
\hline $\mathrm{SRO} 05^{\mathrm{a}}$ & Fox & Adult worm & Intestine & Necropsy & $\begin{array}{l}\text { Mesocestoides } \\
\text { lineatus }\end{array}$ & Košice & Košice & $\begin{array}{l}\text { Slovak } \\
\text { Republic }\end{array}$ & MH463516 & na \\
\hline
\end{tabular}

Deposited in the Parasitic Worms Collection at the Natural History Museum, London under the accession numbers BMNH 2011.2.2.1-3 and BMNH 2011.2.2.19-20. Abbreviation: na not available as not amplified

analysis of 10,000 random quartets was performed using TreePuzzle $5.3[39,40]$. The datasets were used to plot a phylogenetic tree using the maximum likelihood (ML) algorithm implemented in MEGA7 [41] with 1000 bootstrap replications, and the Kimura 2-parameter (K2P) as a molecular evolutionary model. The nodes of the trees with bootstrap values lower than $50 \%$ were considered not well-supported and thus collapsed.
The combined use of two species delimitation methods, the Automatic Barcode Gap Discovery (ABGD) [42] and the Nucleotide Divergence Threshold (NDT) [42], allowed us to make inferences on the occurrence of taxonomic entities by means of two alternative distance models (simple p-distance for ABGD and Kimura (K80) distance for NDT). ABGD was calculated by means of the ABGD online tool (available at http://wwwabi.snv.jussieu.fr/public/ 


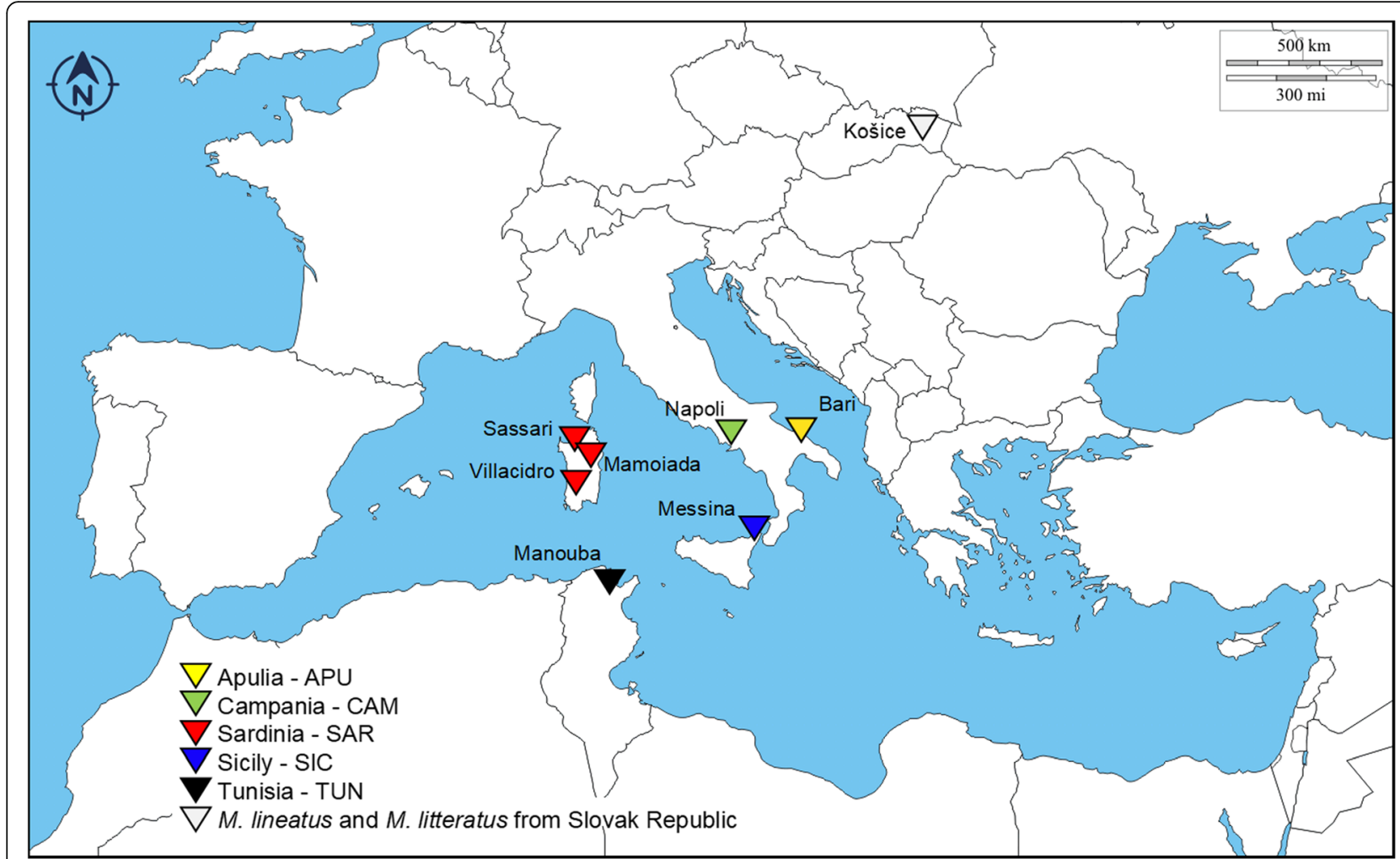

Fig. 1 Map of the Mediterranean indicating the sampling sites

abgd/abgdweb.html) with a prior $P$ ranging from 0.001 to 0.12 , steps $=10$ and relative gap width $(\mathrm{X})=1$. The NDT method was applied by means of a script written in the $\mathrm{R}$ statistical environment (available at https://cran.r-project.org/) and described in [43-45]. Estimates of evolutionary divergence over sequence pairs between groups were conducted in MEGA7 to evaluate the genetic distance between taxa by using the Kimura 2-parameter model.

\section{Results}

The correct taxonomic attribution of specimens from Slovak Republic used as comparative material in the present study, was verified via a BLASTsearch against the available data in the GenBank nucleotide database. They were attributed to M. litteratus and M. lineatus respectively (see Table 1 for details on species and GenBank accession numbers). The analysis of the $\operatorname{cox} 1$ dataset evidenced two haplotypes for M. litteratus $(n=$ $3, \mathrm{~S}=2, \mathrm{~h}=0.667, \pi=0.00660$ ) and one haplotype for $M$. lineatus $(n=1)$. The analysis of the nad 1 dataset evidenced two haplotypes for M. litteratus $(n=3, \mathrm{~S}=3$, h $=0.667, \pi=0.00536$ ) and one haplotype for $M$. lineatus $(n=2)$. The phylogenetic analysis below reported for the Mediterranean region corroborated the taxonomic attribution of specimens from Slovak Republic.

Overall, high levels of genetic variation were found for the cox 1 dataset (373 bp long) among 21 Mesocestoides
Table 2 Sample sizes and genetic diversity estimates obtained for the mitochondrial regions, cox1 (378 bp) and nad1 (558 bp). Sites with gaps were not considered. Sample codes are listed in Table 1

\begin{tabular}{llllll}
\hline Sample & $\mathrm{n}$ & $\mathrm{S}$ & $\mathrm{H}$ & $\mathrm{h}$ & $\pi$ \\
\hline cox1 & 8 & 37 & 5 & 0.857 & 0.03380 \\
Apulia & 1 & 0 & 1 & 0.000 & 0.00000 \\
Campania & 3 & 37 & 3 & 1.000 & 0.06613 \\
Sardinia & 3 & 42 & 3 & 1.000 & 0.07685 \\
Sicily & 6 & 15 & 4 & 0.800 & 0.02377 \\
Tunisia & 21 & 63 & 11 & 0.914 & 0.04708 \\
Total & & & & & \\
nad1 & 8 & 24 & 4 & 0.750 & 0.01773 \\
Apulia & 1 & 0 & 1 & 0.000 & 0.00000 \\
Campania & 2 & 68 & 2 & 1.000 & 0.12186 \\
Sardinia & 3 & 78 & 3 & 1.000 & 0.09550 \\
Sicily & 3 & 3 & 2 & 0.667 & 0.00358 \\
Tunisia & 17 & 82 & 9 & 0.897 & 0.04502 \\
Total & & & & & \\
\hline
\end{tabular}

Abbreviations: $n$ sample size; $S$ number of polymorphic sites, $H$ number of haplotypes, $h$ haplotype diversity, $\pi$ nucleotide diversity 
specimens from the Mediterranean region, with rather low indices of genetic divergence found for the samples from Tunisia ( $h=0.800, \pi=0.024)$ (see Table 2 for estimates of genetic divergence).

Four cox 1 haplotypes were shared by $67 \%$ of the samples while the remaining haplotypes were unique to single individuals (see Additional file 1: Table S1). Median-joining network analysis revealed the occurrence of three main divergent groups of haplotypes (N1, N2 and N3) (see Fig. 2a for details on the geographical distribution of haplotypes). Statistical parsimony network analysis revealed four disconnected clusters within Mediterranean Mesocestoides specimens (Fig. 2b). Three of these clusters ( $\alpha, \beta$ and $\gamma$ ) exactly matched the groups of haplotypes in the median-joining network (N1, N2 and N3, respectively). The highest root weight was shown by a haplotype found in Sardinia (SAR01) for cluster $\alpha$, by haplotypes found in Tunisia and Apulia (TUN03-05, APU04) for cluster $\beta$, and by a haplotype found in Tunisia (TUN02) for cluster $\gamma$. The Bayesian model-based clustering implemented in Baps 6 identified four distinct groups of haplotypes (B1, B2, B3 and B4) (see Fig. 3a for details on the geographical distribution of groups). B2 was the least frequent group, being only reported for the highly divergent haplotype from Apulia (APU08).

A 555 bp long alignment for the nad 1 gene included sequences belonging to 17 Mesocestoides specimens from Italy and Tunisia (Table 1). As a possible consequence of a limited homology between universal primers used and the annealing region, scorable $n a d 1$ sequences were obtained for a reduced number of individuals. Overall, high levels

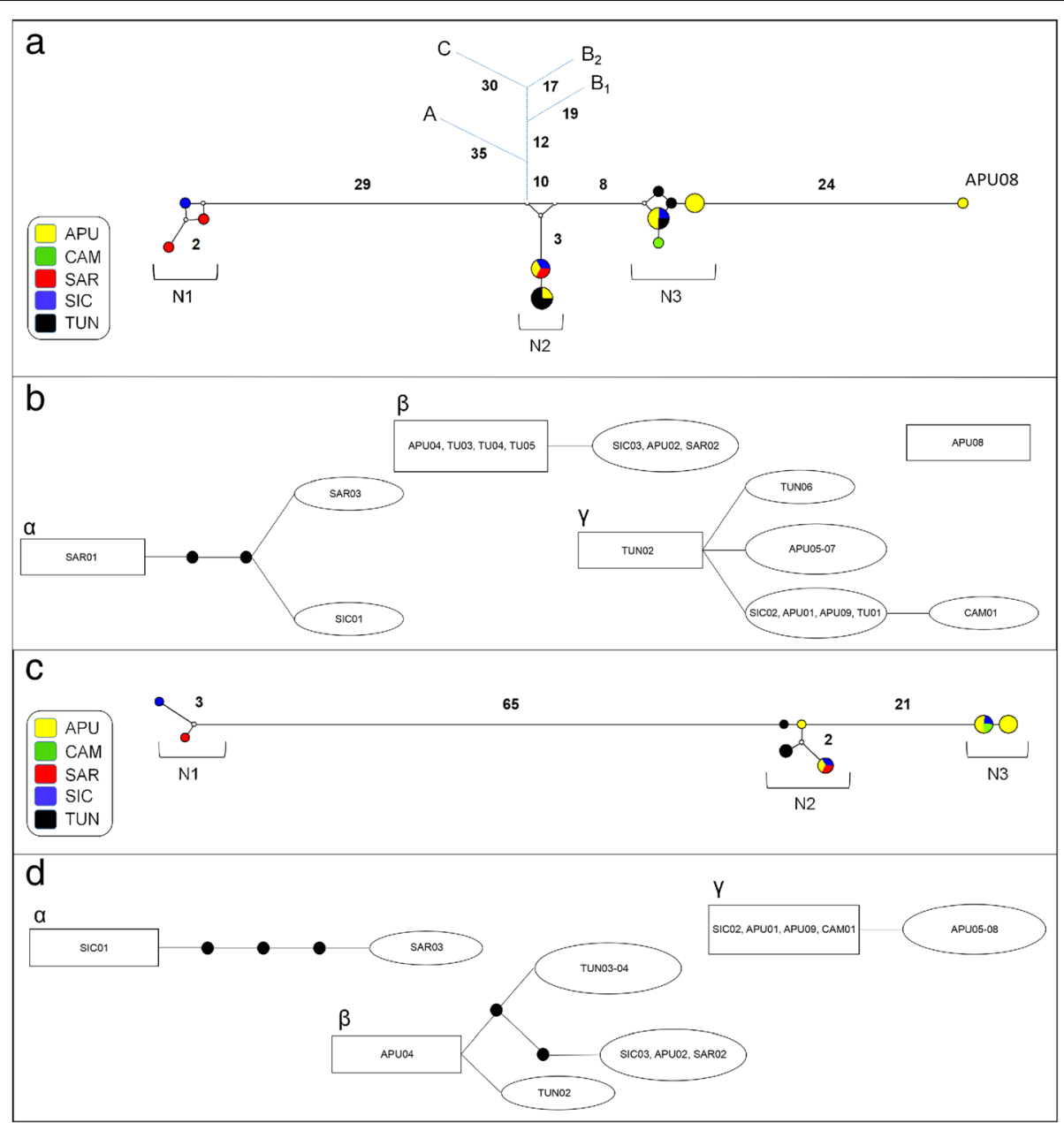

Fig. 2 Network analysis. a, b cox 1 dataset; c, d nad 1 dataset. a, c Median-joining networks with haplotypes coloured according to their geographical distribution. Small white dots on the nodes show median vectors representing hypothetical connecting sequences, calculated with a maximum parsimony method. The numbers of mutations between haplotypes greater than one are reported on the network branches. In the median-joining networks based on cox 1 dataset (a) the short blue branches represent the connection with the other species. Abbreviations: A, Mesocestoides litteratus; $B_{1}$ and $B_{2}, M$. lineatus from Mongolia and Slovak Republic, respectively; $C, M$. corti. b, d Clusters retrieved using 95\% statistical parsimony networks. The number of mutations greater than one are shown as black dots on the network branches. The haplotype in a square has the largest outgroup weight. Abbreviations: APU, Apulia; CAM, Campania; SAR, Sardinia; SIC, Sicily; TUN, Tunisia 

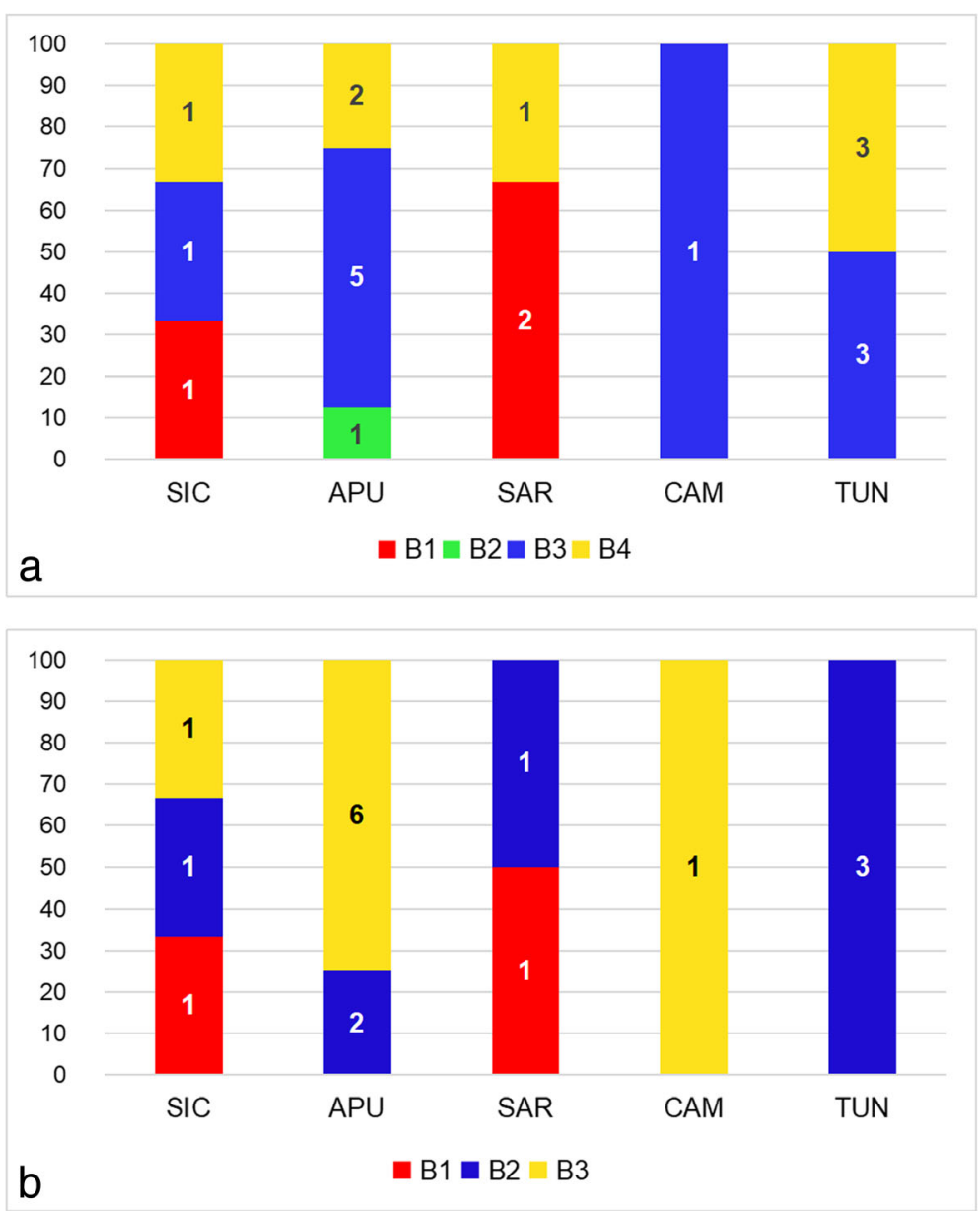

Fig. 3 Distribution of the groups identified by Bayesian model-based clustering implemented in Baps 6 within populations. a cox 1 dataset. b nad1 dataset. $X$ axis: populations; $Y$ axis: relative frequency of distribution (\%). Abbreviations: APU, Apulia; CAM, Campania; SAR, Sardinia; SIC, Sicily; TUN, Tunisia. The numbers in bars indicate the absolute frequency of distribution. B1, B2, B3 and B4 indicate the groups identified by Bayesian model-based clustering described in the text

of genetic variation were found in the Mediterranean region, which resulted in a total genetic variability similar to that found for the cox1 dataset (see Table 2 for details). Three nad 1 haplotypes were found in $65 \%$ of the samples, while the remaining were unique to single individuals (see Additional file 2: Table S2 for more details). The medianjoining network analysis revealed the occurrence of three groups of haplotypes (N1, N2 and N3) (see Fig. 2c for details) almost corresponding to those found for the $\operatorname{cox} 1$ median-joining network analysis (see Fig. 1a). Accordingly, the statistical parsimony network analysis highlighted the occurrence of three disconnected clusters $(\alpha, \beta$ and $\gamma$ ) within Mediterranean Mesocestoides spp. (see Fig. 2d). The highest root weight was shown by a haplotype found in $\mathrm{Si}$ cily (SIC01) for cluster $\alpha$; by a haplotype found in Apulia (APU04) for cluster $\beta$; and by haplotypes found in Apulia (APU01, APU09), Campania (CAM01) and Sicily (SIC02) for cluster $\gamma$.
The Bayesian model-based clustering implemented in Baps 6 (see Fig. 3b for details) identified three groups of haplotypes (B1, B2 and B3) which were consistent with three (B1, B3 and B4) of the four groups reported for the cox1 Bayesian analysis.

The likelihood map based on cox1 gene dataset (see Additional file 3: Figure S1a) indicated a strong phylogenetic signal. The maximum likelihood (ML) tree analysis (Fig. 4) showed five supported and one unsupported (bootstrap value of $45 \%$ ) clusters; three for $M$. litteratus, $M$. corti/M. vogae and $M$. lineatus, and the remaining for the Mediterranean Mesocestoides spp. specimens analysed in the present study. Notably, the $M$. lineatus cluster included a GenBank sequence (KU821650) from Sicily. The individuals of Mesocestoides spp. examined here grouped into three different clusters (M1, M2 and M3 in Fig. 4). A consensus sequence of Mesocestoides sp., from GenBank (JQ740884) from Tuscany, Italy, was also included in the M3 group. 


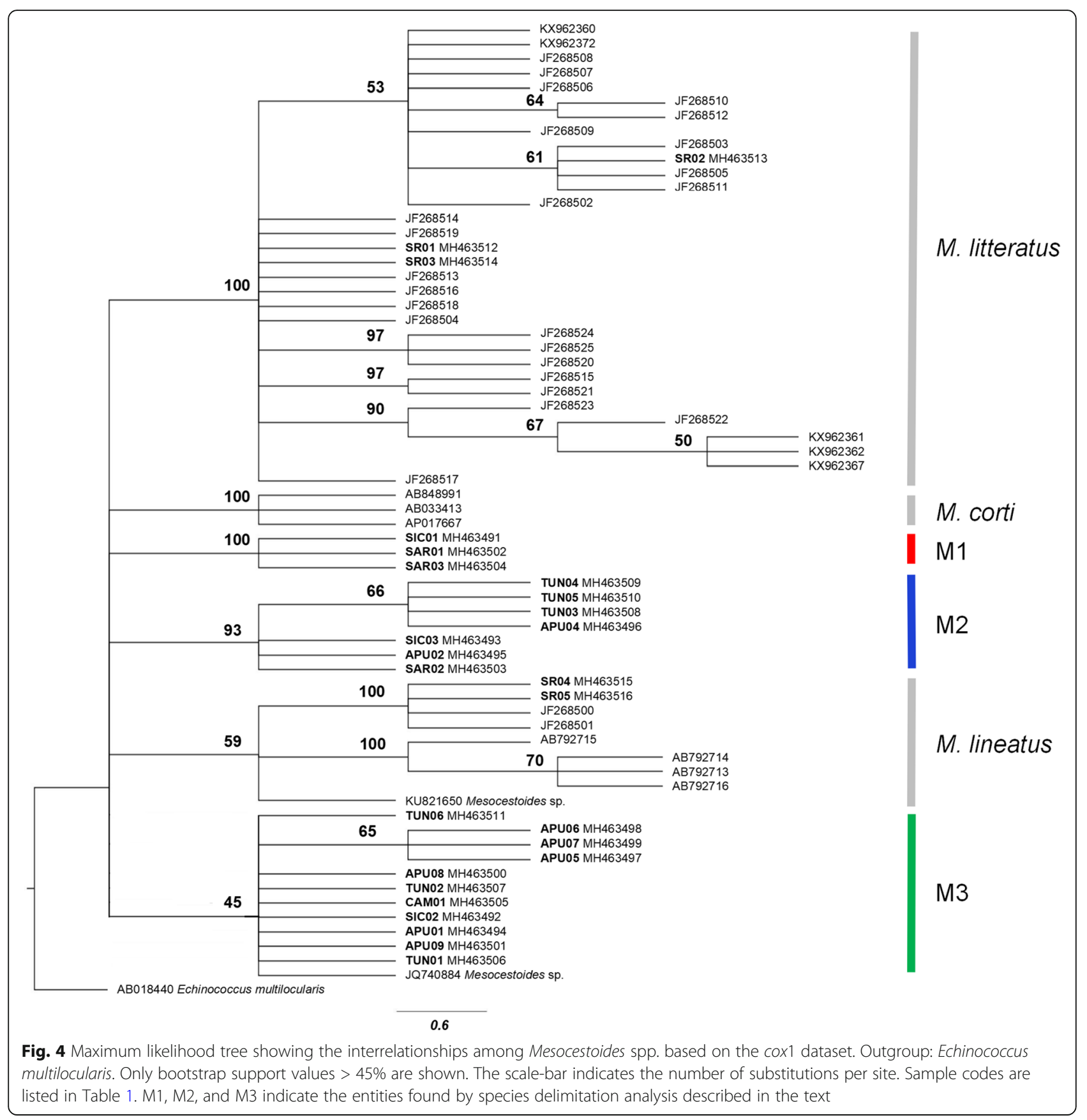

The species delimitation methods, Automatic Barcode Gap Discovery (ABGD) and Nucleotide Divergence Threshold (NDT) converged on the same results; for this reason, only the ABGD results are reported. Four entities were identified within the Mediterranean Mesocestoides spp. analysed. Overall, the composition of all the groups found by ABGD matched the clusters in the ML tree analysis. Evolutionary divergences were estimated between the ML clusters of Mesocestoides spp. (M. litteratus, M.corti/M. vogae, M. lineatus, M1, M2 and M3) (see Additional file 4: Table S3 for details). Consistent with the previous analysis which converged in separating APU08 from the remaining samples, this specimen was considered as a further separate group to be tested.

The likelihood map based on the nad1 gene (see Additional file 3: Figure S1b) indicated a low phylogenetic signal. The ML tree analysis was consistent in showing the same results for the cox 1 dataset (see Fig. 5 for a comparison). Additionally, the species delimitation methods (ABGD and NDT) converged on the same results, and the composition of the groups obtained exactly matched the clusters obtained by ML tree analysis. The ABGD method identified 


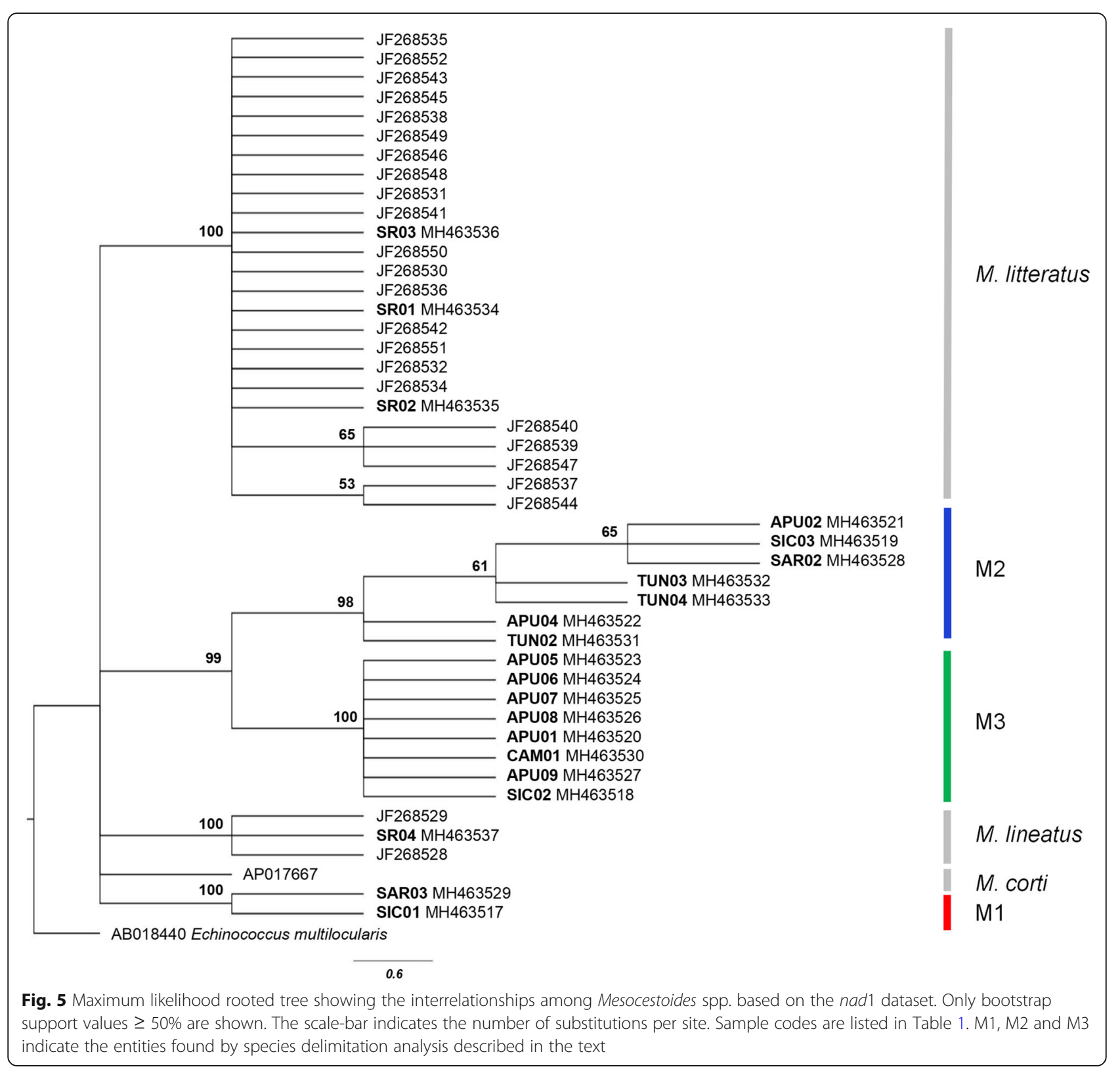

three entities within the Mediterranean Mesocestoides spp. analysed, with the position of the individual from Tunisia TUN02 representing the only discrepancy between the cox 1 and nad 1 ML tree and species delimitation analysis. Estimates of evolutionary divergences for $n a d 1$ gene are reported in the Additional file 5: Table S4.

\section{Discussion}

This study on mitochondrial genetic variability of Mesocestoides spp. from domestic and wild carnivores in the Mediterranean area allowed us to gather a deep definition of the species delimitation among samples from southern Italy and Tunisia. Indeed, molecular analyses were consistent in pointing out three, well-defined, taxonomic Mesocestoides entities from Italy and Tunisia with high levels of genetic variation among individuals and no evidence of geographical structuring among entities (namely M1, M2 and M3 as in ML analysis). The occurrence of a sequence (GenBank: JQ740884) from Tuscany belonging to the M3 entity suggests that the range of distribution of these parasites probably extends north and not only confined to the Mediterranean region. Such a finding is consistent with a study [46], which highlighted the occurrence of a species genetically divergent from M. lineatus, M. litteratus and M. corti, in northern Italy. Two of the Mesocestoides entities in the present study (M2 and M3) displayed very low levels of genetic divergence among each other; the nad1 results evidenced 
the occurrence of a reciprocal monophyly between them, likely consistent with the presence of two Mesocestoides sister taxa in the Mediterranean area.

The statistical parsimony network analysis based on the cox 1 dataset suggests that M2 and M3 probably originated in Tunisia, with an ancestor haplotype also present in Apulia. These findings could be consistent with the occurrence of early polymorphisms, maybe common to the southern Italy and Tunisia, possibly due to the translocation of domestic dogs since ancient times.

The levels of genetic divergence found between the Mediterranean Mesocestoides entities and M. lineatus, higher than those found between the M. lineatus geographical internal subgroups from Slovak Republic, Mongolia and Italy, further support the occurrence, at least in Italy and Tunisia, of Mesocestoides entities strongly divergent from $M$. lineatus. Notably the latter, which is described in the Italian Peninsula [2], was not identified in this work. Conversely, M. lineatus has been previously identified in Italy in a cat from Sicily [27], as evidenced by sequence GenBank: KU821650 included as an outlier within the $M$. lineatus cluster. This finding suggests that possibly several Mesocestoides spp. may occur in sympatry in southern Italy.

From a systematic perspective, the co-occurrence of different molecular entities, does not allow for unequivocal identification and description of the new taxa corresponding to the entities found. Furthermore, the inconsistency between morphological and molecular features supports the hypothesis that different environmental and ecological features interacted in the Mediterranean area to produce cryptic species within the genus Mesocestoides that are genetically divergent but morphologically indistinguishable from each other. In this context, it is important to underline the pivotal role of the molecular taxonomy, not only in identifying the cut-off to delimit species from each other, but also to the naming of the species itself, which result from the validation of the primary species hypotheses [47-53]. In fact, from a viewpoint that takes into account the appreciation of specific biodiversity, no species can be documented without a formal description, as well as no OTUs may substitute a species in any species checklist. [28, 54]. For this reason, the combined analysis of molecular and morphological data, in the light of the "integrative taxonomy approach" [55] will be used in the near future to provide both a satisfactory insight on the evolutionary processes and taxonomic richness, with a formal description of new species of the genus Mesocestoides in the Mediterranean area.

\section{Conclusions}

The present study represents the first survey on mitochondrial genetic variability of Mesocestoides spp. from domestic and wild carnivores in the Mediterranean area that allowed to point out three defined, taxonomic
Mesocestoides entities which are genetically divergent from $M$. lineatus, $M$. litteratus and $M$. corti.

\section{Additional files}

Additional file 1: Table S1. Distribution (absolute frequencies) of cox1 haplotypes in 21 specimens from five Mediterranean sites. Sample codes are listed in Table 1. (DOCX $15 \mathrm{~kb}$ )

Additional file 2: Table S2. Distribution (absolute frequencies) of nad1 haplotypes in 17 specimens from five Mediterranean sites. Sample codes are listed in Table 1. (DOCX $15 \mathrm{~kb}$ )

Additional file 3: Figure S1. Likelihood mapping. a cox1 dataset. b nad 1 dataset. For both panels: (i) distribution map of dots $P$, where $P$ represents the likelihoods of the three possible unrooted trees for a set of four sequences (quartets) [54]. Dots close to the corners and to the sides represent tree-like and network-like phylogenetic signal. Central area represents star-like signal (phylogenetic noise); (ii) percentage distribution of the three possible unrooted trees; (iii) partitions of the area of the triangle into seven regions. The three trapezoids at the corners represent the areas supporting strictly bifurcating trees, that is the presence of a tree-like phylogenetic signal. The three rectangles on the sides represent regions where the decision between two trees is not obvious. The centre of the triangle represents sets of points $\mathrm{P}$ where all three trees are equally supported. (TIF $5240 \mathrm{~kb}$ )

Additional file 4: Table S3. Estimates of evolutionary divergence over sequence pairs between groups based on the cox 1 dataset. Genetic distances, represented by the number of base substitutions per site from averaging over all sequence pairs between groups, are shown below the diagonal and standard deviations above the diagonal. Analyses were conducted using the K2P model. Sample codes are listed in Table 1. (DOCX $17 \mathrm{~kb}$ )

Additional file 5: Table S4. Estimates of evolutionary divergence over sequence pairs between groups based on the nad 1 dataset. Genetic distances, represented by the number of base substitutions per site from averaging overall sequence pairs between groups, are shown below the diagonal and standard deviations are shown above the diagonal. Analyses were conducted using the K2P model. Sample codes are listed in Table 1. (DOCX $18 \mathrm{~kb})$

\section{Abbreviations}

ML: Maximum likelihood; ABGD: Automatic Barcode Gap Discovery; NDT: Nucleotide Divergence Threshold

\section{Acknowledgments}

The authors would like to thank R. P. Lia, V. Tilocca, L. Rinaldi, P. Cabras and B. Boufana, for providing Mesocestoides spp. samples.

\section{Funding}

Not applicable

\section{Availability of data and materials}

All relevant data are within the paper and its additional files. Nucleotide sequences obtained in this study are available in the GenBank database under the accession numbers MH463491-MH463537. Fragments from five adult individuals of $M$. litteratus and one of $M$. lineatus found in foxes from Slovak Republic were deposited in the Parasitic Worms Collection at the Natural History Museum, London under the accession numbers BMNH 2011.2.2.1-3 and BMNH 2011.2.2.19-20.

\section{Authors' contributions}

Conceived and designed the experiments: AV. Performed the experiments: GD and APP. Analyzed the data: DS. Contributed reagents/materials/analysis tools: GD, APP and DS. Wrote the paper: AV, DS, MC, AS and DO. Collected biological samples: CT, GG, GH and SL. Revised the manuscript: AV, DS, MC, AS and DO. All authors read and approved the final manuscript. 


\section{Ethics approval and consent to participate}

This study was performed following the recommendations of European Council Directive (86/609/EEC) on the protection of animals.

\section{Consent for publication}

Not applicable.

\section{Competing interests}

The authors declare that they have no competing interests.

\section{Publisher's Note}

Springer Nature remains neutral with regard to jurisdictional claims in published maps and institutional affiliations.

\begin{abstract}
Author details
${ }^{1}$ Dipartimento di Medicina Veterinaria, Università di Sassari, Sassari, Italy. ${ }^{2}$ Dipartimento di Scienze Biomediche, Università di Sassari, Sassari, Italy. ${ }^{3}$ National School of Veterinary Medicine, Laboratory of Parasitology, Sid Thabet, Tunisia. ${ }^{4}$ Dipartimento di Scienze Veterinarie, Università di Messina, Messina, Italy. ${ }^{5}$ Institute of Parasitology, Slovak Academy of Sciences, Košice, Slovak Republic. ${ }^{6}$ Dipartimento di Medicina Veterinaria, Università di Bari, Bari, Italy.
\end{abstract}

Received: 14 June 2018 Accepted: 6 November 2018 Published online: 04 December 2018

\section{References}

1. Loos-Frank B. One or two intermediate hosts in the life cycle of Mesocestoides (Cyclophyllidae, Mesocestoididae)? Parasitol Res. 1991;77:726-8.

2. Papini R, Matteini A, Bandinelli P, Pampurini F, Mancianti F. Effectiveness of praziquantel for treatment of peritoneal larval cestodiasis in dogs: a case report. Vet Parasitol. 2010;170:158-61.

3. McAllister CT, Bruce Conn D, Freed PS, Burdick DA. A new host and locality record for Mesocestoides sp. tetrathyridia (Cestoidea: Cyclophyllidea), with a summary of the genus from snakes of the world. J Parasitol. 1991;77:329-31.

4. Literák I, Olson PD, Georgiev BB, Špakulová M. First record of metacestodes of Mesocestoides sp. in the common starling (Sturnus vulgaris) in Europe, with an 18S rDNA characterization of the isolate. Folia Parasitol. 2004;51:45-9.

5. Hrčkova G, Miterpáková M, O'Connor A, Šnábel V, Olson P. Molecular and morphological circumscription of Mesocestoides tapeworms from red foxes (Vulpes vulpes) in central Europe. Parasitology. 2011;138:638-47.

6. Lahmar S, Boufana B, Ben Boubaker S, Landolsi F. Intestinal helminths of golden jackals and red foxes from Tunisia. Vet Parasitol. 2014;204:297-303.

7. Dalimi A, Sattari A, Motamedi G. A study on intestinal helminthes of dogs, foxes and jackals in the western part of Iran. Vet Parasitol. 2006;142:129-33.

8. Calvete C, Lucientes J, Castillo JA, Estrada R, Gracia MJ, Peribáñez MA, Ferrer M. Gastrointestinal helminth parasites in stray cats from the mid-Ebro Valley, Spain. Vet Parasitol. 1998;75:235-40.

9. Karamon J, Dabrowska J, Kochanowski M, Samorek-Pieróg M, Sroka J, Różycki M, Bilska-Zajac E, Zdybel J, Cencek T. Prevalence of intestinal helminths of red foxes (Vulpes vulpes) in central Europe (Poland): a significant zoonotic threat. Parasit Vectors. 2018;11:436.

10. Crosbie PR, Boyce WM, Platzer EG, Nadler SA, Kerner C. Diagnostic procedures and treatment of eleven dogs with peritoneal infections caused by Mesocestoides spp. J Am Vet Med Assoc. 1998;213:1578-83.

11. Siles-Lucas M, Hemphill A. Cestode parasites: application of in vivo and in vitro models for studies on the host-parasite relationship. Adv Parasitol. 2002;51:133-230.

12. Boyce W, Shender L, Schultz L, Vickers W, Johnson C, Ziccardi M, et al. Survival analysis of dogs diagnosed with canine peritoneal larval cestodiasis (Mesocestoides spp.). Vet Parasitol. 2011;180:256-61.

13. Montalbano Di Filippo M, Meoli R, Cavallero S, Eleni C, De Liberato C, Berrilli F. Molecular identification of Mesocestoides sp. metacestodes in a captive goldhanded tamarin (Saguinus midas). Infect Genet Evol. 2018;65:399-405.

14. Fuentes MV, Galán-Puchades T, Malone JB. A new case report of human Mesocestoides infection in the United States. Am J Trop Med Hyg. 2003;68: 566-7.

15. Széll Z, Tolnai Z, Sréter T. Environmental determinants of the spatial distribution of Mesocestoides spp. and sensitivity of flotation method for the diagnosis of mesocestoidosis. Vet Parasitol. 2015;212:427-30.
16. Zaleśny G, Hildebrand J. Molecular identification of Mesocestoides spp. from intermediate hosts (rodents) in central Europe (Poland). Parasitol Res. 2012; 110:1055-61.

17. Tenora F. Mesocestoides litteratus (Batsch, 1786) (Cestoda), parasite of Vulpes vulpes (L., 1758) (Carnivora) in the Czech Republic. Acta Univ Agric Silvic Mendel Brun. 2005;53:185-8.

18. Skrjabin Kl. Tetrabotrididae and Mesocestoididae - platyhelminths of birds and mammals. In: Skrjabin Kl, editor. Osnovy Cestodologii, Volume 9. Moscow: Nauka; 1978. p. 156-215. (In Russian).

19. Jančev J. Morphology, taxonomy and distribution of the species of genus Mesocestoides Vaillant, 1863 in Bulgaria. Khelmintologiya. 1986;21:45-65.

20. De Jong $Y$, Verbeek M, Michelsen V, Bjørn PP, Los W, Steeman F, et al. Fauna Europaea - all European animal species on the web. Biodivers Data J. 2014;2:e4034.

21. Andras T, Peter T. Data on worm infestation cats (Felis catus) in Hungarian hunting areas. Magy Allatorvosok Lapja. 2002;124:26-30.

22. Segovia JM, Guerrero R, Torres J, Miguel J, Feliu C. Ecological analyses of the intestinal helminth communities of the wolf, Canis lupus, in Spain. Folia Parasitol. 2003;50:231-6.

23. Martinez-Carrasco C, Ruiz De Ybanez MR, Sagarminaga JL, Garijo MM, Moreno F, Acosta I, et al. Parasites of the red fox (Vulpes vulpes Linneus, 1758) in Murcia, southeast Spain. Rev Med Vet. 2007;158:331-5.

24. Krone O, Guminsky O, Meinig H, Hermann M, Trinzen M, Wibbelt G. Endoparasite spectrum of wild cats (Felis silvestris Schreber, 1777) and domestic cats (Felis catus L.) from the Eifel, Pfalz region and Saarland, Germany. Eur J Wildl Res. 2008;54:95-100.

25. Magi M, Macchioni F, Dell'Omodarme M, Prati MC, Calderini P, Gabrielli S, et al. Endoparasites of red fox (Vulpes vulpes) in central Italy. J Wildl Dis. 2009:45:881-5.

26. Bonfanti U, Bertazzolo W, Pagliaro L, Demarco B, Venco L, Casiraghi M, Bandi C. Clinical, cytological and molecular evidence of Mesocestoides sp. infection in a dog from Italy. J Vet Med A Physiol Pathol Clin Med. 2004;51: 435-8.

27. Lanteri G, Di Caro G, Capucchio MT, Gaglio G, Reina V, Lo Giudice C, Zanet S, Marino F. Mesocestoidosis and multivisceral tetrathyridiosis in a European cat. Vet Med Czech. 2017;62:356-62.

28. Otranto D, Varcasia A, Solinas C, Scala A, Brianti E, Dantas-Torres F, et al. Redescription of Cercopithifilaria bainae Almeida \& Vicente, 1984 (Spirurida, Onchocercidae) from a dog in Sardinia, Italy. Parasit Vectors. 2013;6:132.

29. Littlewood DTJ, Waeschenbach A, Nikolov PN. In search of mitochondrial markers for resolving the phylogeny of cyclophyllidea tapeworms (Platyhelminthes, Cestoda) - a test study with Davaineidae. Acta Parasitol. 2008:53:133-44.

30. Sanna D, Dedola GL, Lai T, Curini-Galletti M, Casu M. PCR-RFLP: a practical method for the identification of specimens of Patella ulyssiponensis s.l. (Gastropoda: Patellidae). Ital J Zool. 2012;79:50-9.

31. Hall TA. BioEdit: A user-friendly biological sequence alignment editor and analysis program for Windows 95/98/NT. Nucleic Acids Symp Ser. 1999;41: 95-8.

32. Librado P, Rozas J. DnaSP v5: a software for comprehensive analysis of DNA polymorphism data. Bioinformatics. 2009;25:1451-2.

33. Bandelt HJ, Forster $P$, Rohl A. Median-joining networks for inferring intraspecific phylogenies. Mol Biol Evol. 1999;16:37-48.

34. Clement M, Posada D, Crandall K. TCS: a computer program to estimate gene genealogies. Mol Ecol. 2000;9:1657-60.

35. Corander J, Cheng L, Marttinen P, Sirén J, Tang JBAPS. Bayesian analysis of population structure V. 6.0. Department of Mathematics and statistics. Helsinki: University of Helsinki; 2013.

36. Kashiide T, Matsumoto J, Yamaya Y, Uwasawa A, Miyoshi A, Yamada K, et al. Case report: first confirmed case of canine peritoneal larval cestodiasis caused by Mesocestoides vogae (syn. M. corti) in Japan. Vet Parasitol. 2014; 201:154-7.

37. Lesniak I, Heckmann I, Heitlinger E, Szentiks CA, Nowak C, Harms V, et al. Population expansion and individual age affect endoparasite richness and diversity in a recolonising large carnivore population. Sci Rep. 2017;7:41730.

38. Strimmer K, Von Haeseler A. Quartet puzzling: a quartet maximumlikelihood method for reconstructing tree topologies. Mol Biol Evol. 1996;13: 964-9.

39. Schmidt HA, Strimmer K, Vingron M, von Haeseler A. TREE-PUZZLE: maximum likelihood phylogenetic analysis using quartets and parallel computing. Bioinformatics. 2002;18:502-4. 
40. Schmidt HA, von Haeseler A. Phylogenetic inference using maximum likelihood methods. In: Lemey P, Salemi M, Vandamme AM, editors. The Phylogenetic Handbook. 5th ed. Cambridge: Cambridge University Press; 2012. p. 181-209.

41. Kumar S, Stecher G, Tamura K. MEGA7: Molecular Evolutionary Genetics Analysis Version 7.0 for bigger datasets. Mol Biol Evol. 2016;33:1870-4.

42. Hebert PDN, Cywinska A, Ball SL, deWaard JR. Biological identifications through DNA barcodes. Proc Biol Sci. 2003;270:313-22.

43. Scarpa F, Cossu P, Lai T, Sanna D, Curini-Galletti M, Casu M. Meiofaunal cryptic species challenge species delimitation: the case of the Monocelis lineata (Platyhelminthes: Proseriata) species complex. Contrib Zool. 2016;85: 123-45.

44. Scarpa F, Cossu P, Sanna D, Lai T, Casu M, Curini-Galletti M. New insights on the genus Otoplana Du Plessis, 1889 (Platyhelminthes: Proseriata), with description of two new species from the Canary Islands. Mar Biodivers. 2017. https://doi.org/10.1007/s12526-017-0785-1.

45. Scarpa F, Sanna D, Cossu P, Lai T, Casu M, Curini-Galletti M. How to achieve internal fertilization without a vagina: the study case of the genus Archilina Ax, 1959 (Platyhelminthes, Proseriata) from Canary Islands. Mar Biodivers. (In Press) https://doi.org/10.1007/s12526-018-0890-9.

46. Jabbar A, Papini R, Ferrini N, Gasser RB. Use of a molecular approach for the definitive diagnosis of proliferative larval mesocestoidiasis in a cat. Infect Genet Evol. 2012;12:1377-80

47. Varcasia A, Tamponi C, Tosciri G, Pipia AP, Dore F, Schuster RK, et al. Is the red fox (Vulpes vulpes) a competent definitive host for Taenia multiceps? Parasit Vectors. 2015;8:491.

48. Varcasia A, Canu S, Lightowlers MW, Scala A, Garippa G. Molecular characterization of Echinococcus granulosus strains in Sardinia. Parasitol Res. 2006;98:273-7

49. Saarma U, Jõgisalu I, Moks E, Varcasia A, Lavikainen A, Oksanen A, et al. A novel phylogeny for the genus Echinococcus, based on nuclear data, challenges relationships based on mitochondrial evidence. Parasitology. 2009:136:317-28

50. Varcasia A, Jia WZ, Yan HB, Manunta ML, Pipia AP, Garippa G, et al. Molecular characterization of subcutaneous and muscular coenurosis of goats in United Arab Emirates. Vet Parasitol. 2012;190:604-7.

51. Boufana B, Scala A, Lahmar S, Pointing S, Craig PS, Dessì G, et al. A preliminary investigation into the genetic variation and population structure of Taenia hydatigena from Sardinia, Italy. Vet Parasitol. 2015;214:67-74.

52. Scala A., Pipia AP, Dore F, Sanna G, Tamponi C, Marrosu R, et al. Epidemiological updates and economic losses due to Taenia hydatigena in sheep from Sardinia, Italy. Parasitol Res. 2015;114:3137-43.

53. Kinkar L, Laurimaë T, Simsek S, Balkaya I, Casulli A, Manfredi MT, et al. Highresolution phylogeography of zoonotic tapeworm Echinococcus granulosus sensu stricto genotype G1 with an emphasis on its distribution in Turkey, Italy and Spain. Parasitology. 2016;143:1790-801.

54. Jörger KM, Schrödl M. How to describe a cryptic species? Practical challenges of molecular taxonomy. Front Zool. 2013;10:59.

55. Dayrat B. Toward integrative taxonomy. Biol J Linnean Soc. 2005;85:407-15.

Ready to submit your research? Choose BMC and benefit from:

- fast, convenient online submission

- thorough peer review by experienced researchers in your field

- rapid publication on acceptance

- support for research data, including large and complex data types

- gold Open Access which fosters wider collaboration and increased citations

- maximum visibility for your research: over $100 \mathrm{M}$ website views per year

At BMC, research is always in progress.

Learn more biomedcentral.com/submissions 